\title{
Ultra-light hierarchical meta-materials on a body-centred cubic lattice
}

\author{
Daniel Rayneau-Kirkhope ${ }^{1}$, Yong $\mathrm{Mao}^{2}$, Robert Farr ${ }^{3,4}$ \\ 1 Department of Applied Physics, Aalto University, Espoo, FINLAND \\ 2 School of Physics and Astronomy, University of Nottingham, Nottingham, NG7 2RD, UK \\ 3 Unilever RED, Colworth House, Sharnbrook, Bedford, MK44 $1 L Q$, UK \\ ${ }^{4}$ London Institute for Mathematical Sciences, 35a South Street, Mayfair, London, UK
}

\begin{abstract}
Modern fabrication techniques offer the freedom to design and manufacture structures with complex geometry on many length-scales, offering many potential advantages. For example, fractal/hierarchical struts have been shown to be exceptionally strong and yet light, Phys. Rev. Lett., 109, 204301 (2012). In this letter, we propose a new class of meta-material, constructed from fractal or hierarchical struts linking a specific set of lattice points. We present a mechanical analysis of this meta-material resulting from a body-centred cubic (BCC) lattice. We show that, through the use of hierarchy, the material usage follows an enhanced scaling relation, and both material property and overall efficiency can be optimised for a specific applied stress. Such a design has the potential of providing the next generation of lightweight, buckling-resistant meta-materials.
\end{abstract}

PACS numbers:

The design of strong, light structures is an enduring challenge in human history, driven by economic demands as much as technological possibilities. A dramatic increase in the interest of mechanical meta-materials and "designer matter" has been witnessed in recent years [1-5]. This growth has been stimulated by new fabrication techniques, which have relaxed the constraints on length-scale and geometric complexity to which a designer must adhere. For example, designs with features on the nanometer scale can now be fabricated using techniques such as two-photon lithography [6] and electroless plating [7]. New methods are also being introduced including controlled self assembly of complex thin walled micro-structures [8], these novel techniques may offer even greater freedom in design. This new found freedom has allowed the creation of meta-materials with non-trivial structural elements on multiple lengthscales $[6,7,9-12]$, resulting in architectures with close resemblance to some remarkable geometries found in nature [13].

Many natural structures owe their remarkable mechanical properties as much to their geometry as their constituent material. The presence of non-trivial structural order on many length-scales is a unifying concept in many such architectures. For example, through hierarchy [13], biological structures offer exceptional performance in adhesion [14], fracture toughness [15], elasticity [16], and strength to weight ratios [17]. Although both theoretical [18-22] and experimental [6, 9-12, 23, 24] work has been undertaken, the role of hierarchy in such structures has not yet been fully elucidated: Such understanding will pave the way for new structures and materials that match and exceed the performance of those found in nature.

In this article, we propose a novel hierarchical metamaterial constructed from fractal/hierarchical [25] struts linking a specific set of lattice points. We present a mechanical analysis of the resulting infinite, periodic $3-\mathrm{d}$, pin jointed lattices under arbitrary uniform stress. For this article, we adopt a body-centred cubic lattice, with struts linking nearest and next-nearest lattice sites. In this lattice, all nodes are equivalent, however, our analysis can be generalised to lattices with more than one lattice site in the unit cell. We find that, through prudent choice of the relative spring constant of the linkages within the structure, one can create an isotropic metamaterial. The degree of hierarchy within the linkages may be varied, thus endowing the overall meta-material with a high degree of tailorability in its elastic response. A load can be applied to the meta-material with an arbitrary macroscopic stress tensor, $\sigma_{i j}$, which defines the stress vector $\boldsymbol{\sigma}_{\mathrm{M}} \equiv\left(\sigma_{11}, \sigma_{22}, \sigma_{33}, \sigma_{23}, \sigma_{13}, \sigma_{12}\right)^{T}[26]$. For some magnitude of this loading, the linkages will fail either elastically or through yielding of the material. For a given stress direction $\hat{\boldsymbol{\sigma}}_{M}$, we aim, through hierarchical design, to create meta-materials maintaining elastic stability under a given relative stress $\sigma / \sigma_{Y}$, while minimising the relative density of material, $\rho / \rho_{m}$, where $\rho$ is the density of the meta-material, $\sigma=\left|\boldsymbol{\sigma}_{M}\right|$, and $\sigma_{Y}$ and $\rho_{m}$ are the yield stress and density of the construction material respectively. While it is noted that other modes of failure could occur, our interest here is in metamaterials of low relative density, thus linkages with high aspect ratios are assumed throught, consequently elastic failure is the limiting factor. In order to maximise the robustness of our results, that is, broaden the spectrum of stress directions over which the hierarchical design shows beneficial results, we use a space frame design considered sub-optimal under simple compressive loading as our linkage element; the structure considered here is a modified version of that presented in Ref. [27], where a fractal beam structure was optimised for gentle compressive axial loading. This paper is divided into three sections: In the first section we present the key properties of the hierarchical linkage element used here. In section II, using a novel methodology, we derive the mechanical properties of our chosen pin jointed lattice, establishing the necessary properties of our linkages to give isotropic 

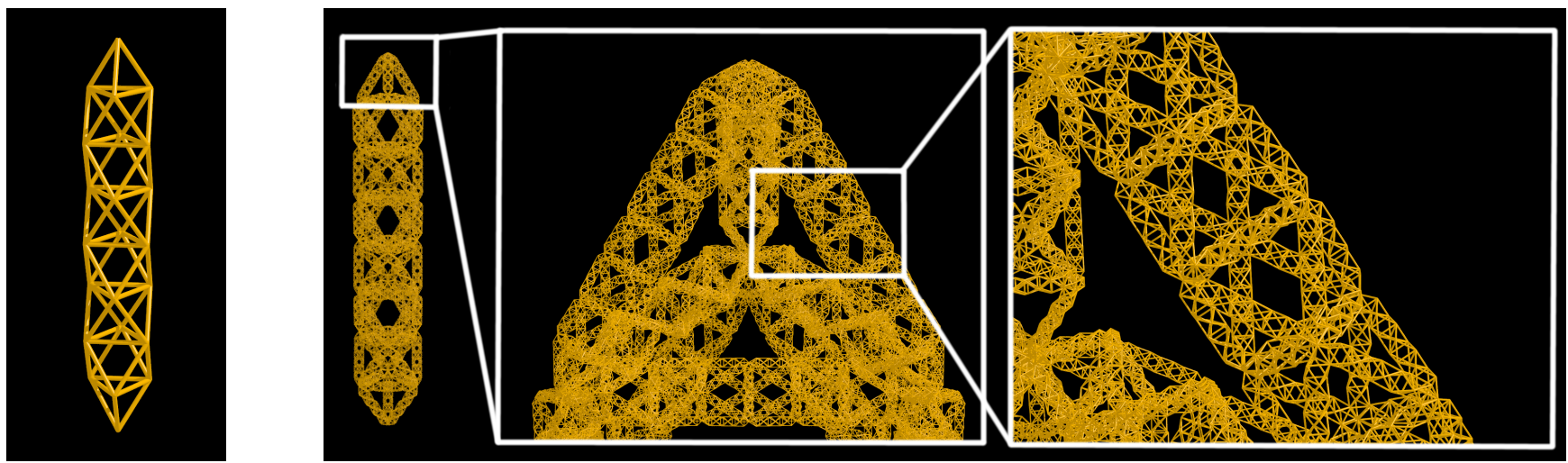

FIG. 1: The hierarchical frame: Left shows generation-1 frame, with $n_{1,1}=6$; The right image shows a generation-3 structure with insets showing details at an increasingly small scale. The particular frame shown is with parameters $n_{3, i}=6$ for $i=1,2,3$. In contrast to [27], in this work, at each iterative step, all beams within the structure are replaced by generation-1 frames.

material properties, and the consequent effective Young's modulus and Poisson's ratio of our meta-material.

In the section III, we combine the results of the first two sections to establish the elastic limit of our lattice based meta-material with hierarchical linkages for various directions of applied stress, showing significant gains through hierarchical design. We show that for low relative density of material, the use of hierarchy becomes increasingly beneficial, and that these gains occur over a wide range of stress orientations. Furthermore, we show that for a given stress direction, a series of scaling relationships exist relating the minimum volume fraction of material, $\rho / \rho_{m}$ and the magnitude of the relative stress that results in elastic failure, $\sigma / \sigma_{Y}$, which depend on the degree of hierarchy of the linkages. For a wide variety of stress directions, these scaling relations can be manipulated in a systematic and beneficial manner.

\section{LINKAGE ELEMENTS}

Simple hollow beams: To serve as a reference for the hierarchical structures, we will consider the lattice structure fabricated from thin walled cylinders. Euler buckling [28] provides a first limit for the loading capacity of such a structure under compression. Assuming freely hinged end points, a slender beam of length $L$ buckles under a force $F$

$$
F=\frac{\pi^{2} Y I}{L^{2}}
$$

where $I$ is the second moment of area which, for a thin walled cylinder, is approximated by $I \approx \pi r^{3} t$, where $r$ is the radius of the beam and $t$ is the wall thickness. In a thin walled structure, a short wavelength buckling can also occur $[29,30]$, providing a second limit to the compressive loading:

$$
F<\frac{2 \pi Y t^{2}}{\sqrt{3\left(1-\nu^{2}\right)}},
$$

where $\nu$ is the Poisson's ratio of the construction material. Utilising these constraints, it is possible to determine the material required for stability of a simple beam for a given value of compressive loading. When the beam is put under tension, the elastic limit of the material requires that the stress does not exceed the yield stress of the material, $\sigma_{Y}$. The thin walled cylinder will be referred to as the generation-1 structure.

Hierarchical elements: The first space frame design (generation-2) that we consider is shown in figure 1 (left), constructed from thin walled beams forming $n_{1,1}$ octahedra linking two end tetrahedra. The higher order structures considered here follow a simple iterative procedure, the generation- $G$ frame is constructed through the replacement of all beams in the generation- $(G-1)$ structure with scaled space frames, the number of octahedra at each iteration is allowed to vary. We follow the notation introduced first in [18] that allows us to refer to characteristics of a structure at a given length scale: $X_{G, i}$ refers to the parameter $X$ at length scale $i$ of $G(i=1$ being the smallest, $i=G$, the longest). Simple geometry gives

$$
L_{G, i}=\sqrt{2 / 3}\left(n_{G, i}+2\right) L_{G, i-1} .
$$

Elastic stability of a generation- $G$ frame can be lost at one of $G+1$ length-scales: on the smallest scale, the thin walled structure can fail due to Koiter buckling (wavelength of order $r$ ), or Euler buckling (of order $L_{G, 1}$ ). Alternatively, the whole frame or any subframe can fail due to an Euler buckling, with wavelength of order $L_{G, i}$, for $2 \leq i \leq G$.

When a compressive (tensile) axial force $F_{G, G}$ is applied to the generation- $G$ structure, a the maximum load experienced by any substructure at level $i$ in the structure will be [27]

$$
\begin{gathered}
F_{G, i}=-\frac{F}{2^{i} 6^{i / 2}} \\
F_{G, i}=\frac{F_{G, G}}{6^{i / 2}},
\end{gathered}
$$


for tension (compression) and compression (tension) bearing beams in the structure respectively. The mechanical properties of the generation- $G$ will be dependent on its geometry and topology. The spring constant, $K_{G, i}$ and bending stiffness, $Y I_{G, i}$ of a frame and its subframes can be calculated as

$$
\begin{aligned}
K_{G, i} & =\frac{36 K_{G, i-1}}{11 n_{G, i}+43}, \\
Y I_{G, i} & =B L_{G, i-1}^{3} K_{G, i-1},
\end{aligned}
$$

where $B$ is a constant [27]. At all hierarchical levels, there is an Euler buckling mode that must be avoided. The constraint in Eq. (1) must be satisfied for all pairs $\left(Y I=Y I_{G, i}, L=L_{G, i}\right), i \leq G$. Furthermore, $F_{G, 1}$ must not exceed the value given in Eq. (2). Given these expressions, and a fixed length of structure we can perform a naive optimisation on the spaceframe for a given applied load [27]: The parameters $r$ and $t$ are set such that the smallest beams in the structure have a coincident bifurcation point due to Koiter and Euler buckling. Utilising these values, we set $n_{G, i}$ from $i=1$ to $i=G$ for minimal material cost retaining elastic stability beyond the point of failure on length scales below.

While other modes of failure are possible, in the limit of interest here (that is lightweight meta-materials), high aspect ratio structures of beneficial, in this limit elastic stability is likely to be the failure mode of interest. Furthermore, it is noted that for the geometry considered here, when tension is applied to the frame of any generation, each hierarchical level will have a sub-frame that withstands a compressive load, the elastic stability of this element thus introduces the active limit on loading.

\section{ELASTIC PROPERTIES OF META-MATERIAL}

Here we present the analysis of the mechanical properties of a particular 3-dimensional lattice. This analysis allows, given a macroscopic stress applied to the meta-material, the computation of the loading on the constituent hierarchical linkage elements. The analysis presented here is readily generalisable to other lattice geometries. Here, we consider an infinite body-centred cubic lattice and place struts with spring constant $k_{1}$ between nearest neighbours and spring constants of $k_{2}$ between next nearest neighbours, see figure 2 . In doing so we create a lattice where all points on the lattice are equivalent. We take $L$ to be the distance between nearest neighbours. If we take one lattice point to be at the origin of Cartesian coordinates we find that nearest neighbours are at points,

$$
\mathbf{x}_{1, \ldots, 8}=L( \pm 1, \pm 1, \pm 1) / \sqrt{3}
$$

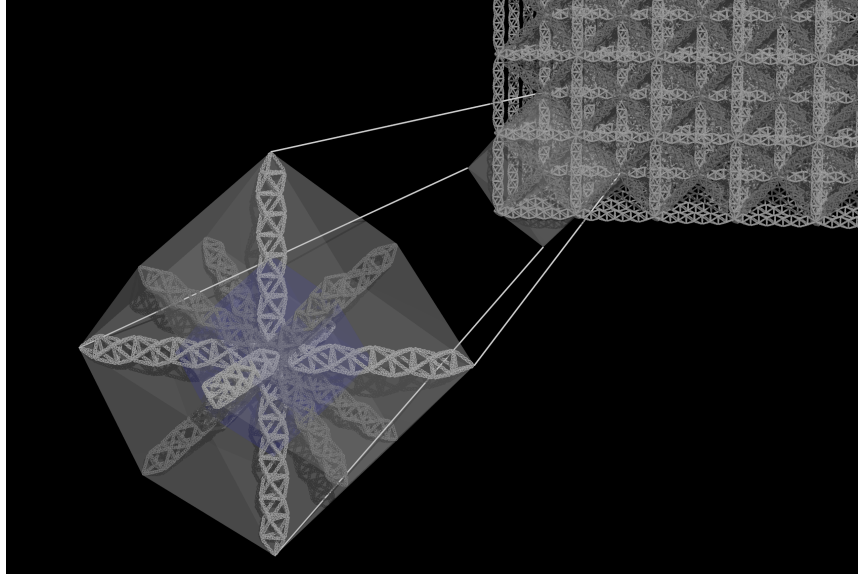

FIG. 2: Showing the body-centred cubic lattice with nearest and next nearest neighbours joined by springs of different stiffness (distinguishable by shade). Also showing a single lattice point with all the attached generation-2 frames considered in the derivation of Eq. (13).

Then, next nearest neighbours are found at:

$$
\begin{aligned}
\mathbf{x}_{9,10} & =2 L( \pm 1,0,0) / \sqrt{3} \\
\mathbf{x}_{11,12} & =2 L(0, \pm 1,0) / \sqrt{3} \\
\mathbf{x}_{13,14} & =2 L(0,0, \pm 1) / \sqrt{3}
\end{aligned}
$$

We follow the Cauchy-Born hypothesis, and consider affine deformations to the lattice structure represented by a symmetric matrix e, we see that under this deformation, a point originally at $\mathbf{x}_{\mathbf{i}}$ is translated to point $\mathbf{x}_{\mathbf{i}}^{\prime}$ given by,

$$
\mathbf{x}_{\mathbf{i}}^{\prime}=\left(\mathbf{I}_{3}+\mathbf{e}\right) \cdot \mathbf{x}_{\mathbf{i}}
$$

where $\mathbf{I}_{\mathbf{3}}$ is the identity matrix. The energy per unit volume can be expressed as a sum over the contributions from each linkage in the unit cell:

$$
U=\frac{1}{2 V_{\mathrm{uc}}} \sum_{i=1}^{14} \frac{1}{2} k_{i}\left[\left|\left(\mathbf{I}_{\mathbf{3}}+\mathbf{e}\right) \cdot \mathbf{x}_{i}\right|-L_{i}\right]^{2}
$$

where $L_{i}$ is the initial distance between the nodes considered, $V_{\mathrm{uc}}$ is the volume of the unit cell, and the factor of half arises to avoid double counting. Through prudent choice of spring constant $k_{1}$ and $k_{2}$ we aim to create an elastically isotropic material. Through equating the Taylor series of Eq. (13), truncated at terms quadratic in e, with the equivalent expression for an isotropic material [31], we find that the relationship

$$
k_{2}=\frac{2 k_{1}}{3}
$$

ensures that the resulting meta-material will be isotropic. Such a meta-material will have Poisson's ratio and 
Young's modulus given by

$$
\tilde{\nu}=\frac{1}{4}, \quad \tilde{Y}=\frac{5 k_{1}}{2 \sqrt{3}} .
$$

Given a macroscopic stress applied to our isotropic material, through the expressions in Eq. (15), we are able to obtain the macroscopic material strain. We now define three vectors that describe the periodicity of the lattice:

$$
\begin{array}{r}
\mathbf{a}_{1}=L(1,1,1)^{T} / \sqrt{3}, \\
\mathbf{a}_{2}=L(-1,1,1)^{T} / \sqrt{3}, \\
\mathbf{a}_{3}=L(1,1,-1)^{T} / \sqrt{3} .
\end{array}
$$

Using these vectors, starting at any point on the lattice a transformation can be found involving integer multiples of these vectors taking us to any equivalent point on the lattice. The strain on the material can then be related to the change in the periodic lattice vectors through $[26]$ :

$$
\Delta \mathbf{a}_{i}=\mathbf{A}\left[\epsilon_{11}, \epsilon_{22}, \epsilon_{33}, \gamma_{23}, \gamma_{13}, \gamma_{12}\right]^{T}
$$

where $\mathbf{A}$ is a matrix whose values depend on the vector $\mathbf{a}_{\mathbf{i}}$ [26]. Thus, for a given stress we can obtain the deformation in each of the space frames that make up our material and consequently the macroscopic failure stress of our meta-material can be found through consideration of the elastic failure loadings of the constituent space frames.

\section{ELASTIC INSTABILITY}

Having derived the material properties of our metamaterial, it is possible to relate macroscopic stress with macroscopic strain. Then, through Eq. (19) we can relate macroscopic strain with the strain (and therefore force, through Eq. (6)) experienced by the member frames. For a given relative density of material, we can then find the failure stress of the meta-material for different degrees of hierarchy. The increase in load bearing capacity of hierarchical structures relative to the generation-1 design are shown in figure 3 for various stress directions and relative densities. It is observed that hierarchy is increasingly beneficial for structures of lower relative density.

Scaling Laws: We further compare the efficiency of meta-materials of various degrees of hierarchy, by establishing the scaling relationship between the minimal value of relative density of material required for stability, and relative stregth of meta-material, the magnitude of stress. For a given generation and loading direction $\hat{\boldsymbol{\sigma}}_{M}$, one can numerically obtain the magnitude of the stress that will result in loss of elastic stability. Scaling laws can then be obtained from these results with a high degree of accuracy.

The scaling relationships observed are dependent on the nature of the stress considered. When compressive load on the linkages causes the dominant mode of failure, for example, the structure is placed under isotropic

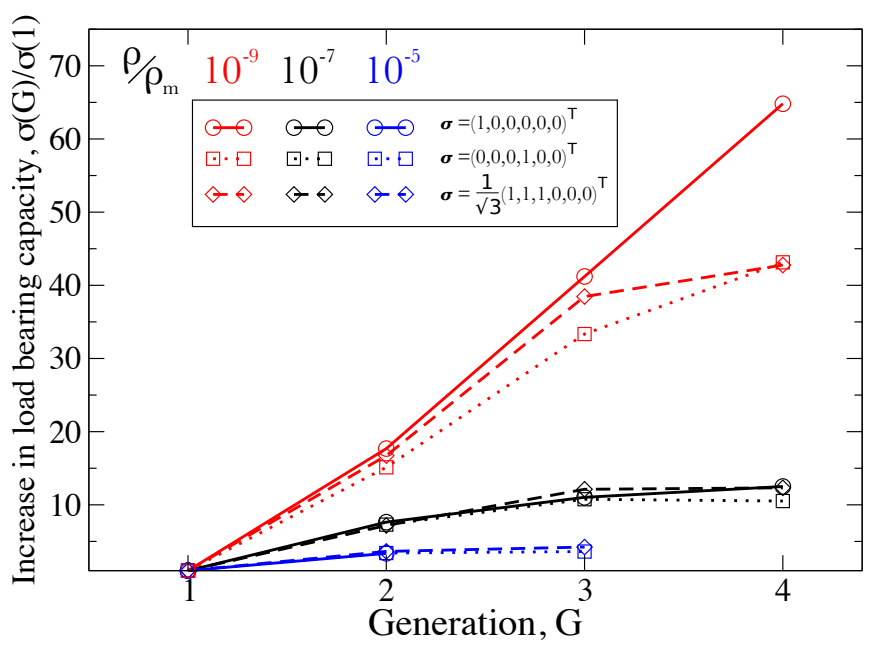

FIG. 3: The increase in maximum stress bearing capacity for different generation of structure, $G$. Here, $\sigma(G)$ is the maximum value of $|\boldsymbol{\sigma}|$ for which a generation- $G$ structure retains elastic stability. Figure shows gains for various relative densities (indicated by color) and loading conditions (indicated by line syle). In all loading conditions shown, the benefits of hierarchical design increase with decreasing relative density of meta-material.

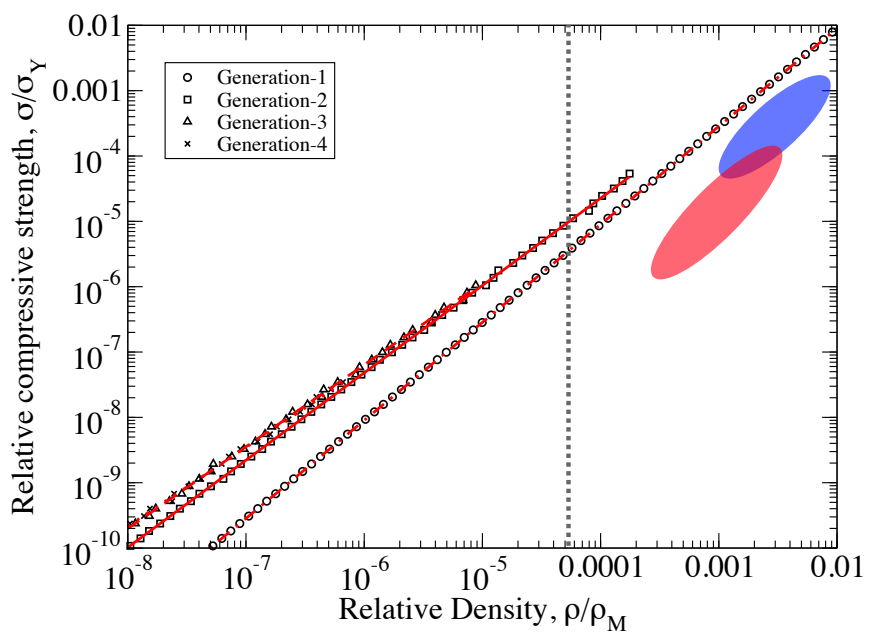

FIG. 4: The relationship between relative density and relative strength under uniaxial compressive loading, material properties are taken to be close to those of $\mathrm{Al}_{2} \mathrm{O}_{3}$ reported in [12]. The red and blue regions show approximate values from experimentally realised octet truss lattice materials of generation- 1 design made from $\mathrm{Al}_{2} \mathrm{O}_{3}$ and Ni-P respectively [12] while the grey dashed line indicates the approximate relative density of an experimentally realised carbon aerogel [32]. The points show the direct results of optimisation (with noise due to the integer nature of $n_{G, i}$ ), while the lines in red show fitted curves, from which the scaling laws (Eq. (20)) are taken with a high degree of accuracy. 

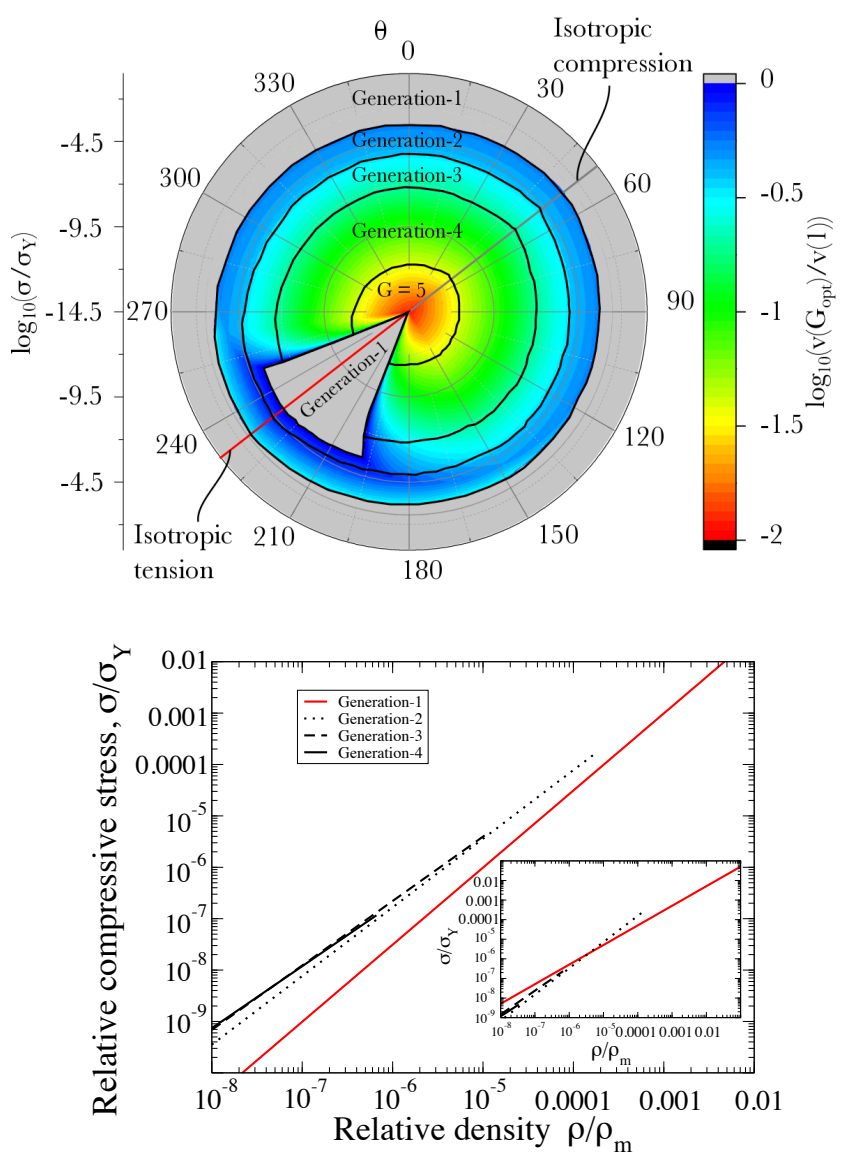

FIG. 5: Above: The optimal generation for applied stress $\boldsymbol{\sigma}_{\mathrm{M}}=\sigma / \sqrt{3} \sin (\theta)[0,-1,-1,-1,0,0]^{T}+$ $\sigma / \sqrt{2} \cos (\theta)[-1,0,0,1,0,0]^{T}$. The construction material properties are assumed to be close to that of $\mathrm{Al}_{2} \mathrm{O}_{3}$, as given in [12]. The stress direction is varied with $\theta$ whose value is constant for a given radial line (indicated in degrees). The distance from the center of the colour map indicates the magnitude of loading, as shown by the scale on the left, while the colour indicates the magnitude of the material saving relative to the generation-1 design. The regions are labeled with the optimal generation number for that direction and magnitude of loading. Below: The scaling relationships for isotropic compression (main figure) and tension (inset), using the same material properties. The only scaling change is observed for generation-1, as described in Eqs. (20 - 21).

crush pressure or uniaxial compressive load, the scaling of relative strength with relative density will follow,

$$
\frac{\sigma}{\sigma_{Y}} \sim\left(\frac{\rho}{\rho_{m}}\right)^{\frac{G+2}{G+1}},
$$

for all values of $G$. These scalings are shown in figure 4, where the efficiency of the structures presented here are compared with existing meta-materials.

If, however, the component frames that make up the material fail under tension (for example isotropic tension), the structure constructed from solid/hollow beams will follow the scaling

$$
\frac{\sigma}{\sigma_{Y}} \sim \begin{cases}\frac{\rho}{\rho_{m}} & \text { if } \quad G=0 \\ \left(\frac{\rho}{\rho_{m}}\right)^{\frac{G+2}{G+1}} & \text { if } \quad G \geq 1\end{cases}
$$

this is shown in the inset of figure 5. It is noted, that in the limit of gentle loading, under compression, higher generation frames will be increasingly efficient, while under tension, simple beams will be optimal.

We can also determine the optimal generation of hierarchical beam for all applied macroscopic stresses. In figure 5 , we show the minimum volume fraction required for stability against a variety of stress directions and magnitudes, alongside the optimal generation number.

\section{DISCUSSION}

We have proposed a novel hierarchical meta-material constructed from fractal/hierarchical struts linking a specific set of lattice points, and we have presented a mechanical analysis of this structure. Using our methodology, we have designed an isotropic meta-material from a body centred cubic lattice with nearest and next nearest neighbours linked with hierarchical beams. Given a component beam of a particular degree of hierarchy, and a general loading stress on the material, we establish the magnitude of loading that will cause elastic instability in the lattice. Through manipulation of hierarchy, we have shown that, for a wide range of loading directions on the material, the fundamental scaling laws defining the efficiency of the meta-material can be manipulated in a beneficial manner. This work illustrates a route to materials with exceptionally high strength to weight ratio.

Harnessing the potential of hierarchical design could provide the next generation of lightweight, functional materials. The increased resolution of modern fabrication techniques have made the use of hierarchical structures such as the one presented here a realistic possibility $[6,11,12]$, and as such the designs presented here are of great economic and technological potential. While the analysis presented here is restricted to beams/frames of uniform construction down their long axis, the analysis can be generalised to more general linkage elements offering further potential for optimisation. It is also noted that the postbuckling behaviour of the meta-material has not yet been elucidated: Understand of this behavour may lead to as yet unanticipated technological applications.

In the future, it may be possible that single and multiwalled carbon nanotubes [33] or DNA helices [35] could act as the component beams on the smallest structural scale, allowing a huge degree of tailorability of the macroscopic material properties. It has been shown that the instability of both carbon nanotubes $[33,34]$ and DNA helices [35-37] structures is broadly similar to those con- 
sidered here, and as such, the analysis presented here

would be expected to hold.

[1] Coulais, C et al., Nature, 535, 529 (2016)

[2] Babaee, S. et al., Adv. Mat., 255044 (2013)

[3] Berger, J. B., et al., Nature, 543, 533 (2017)

[4] Florijn, B. et al., Phys. Rev. Lett., 113, 175503 (2014)

[5] Reis, P. M., et al.,Extreme Mech. Lett., 5, 25 (2015)

[6] Meza, L. R., et al., Proc. Natl Acad. Sci., 112, 11502 (2015)

[7] Schaedler, T. A et al., Science, 334, 934 (2011)

[8] Nadir Kaplan, C., et al., Science 3551395 (2017)

[9] Mousanezhad, D. et al., Sci. Rep., 5, 1038 (2015)

[10] Jang, D., et al., Nat. Mat., 12, 893 (2013)

[11] Zheng X., et al., Nat. Mat., 151100 (2016)

[12] Zheng, X. et al., Science, 3441373 (2014)

[13] Lakes, R., Nature, 511511 (1993)

[14] Gao, H., et al., Mech. Mat., 37275 (2005)

[15] Wegst, U., et al., Nat. Mat., 14, 23 (2015)

[16] Zhou, H., et al., Phys. Rev. Lett., 94028104 (2005)

[17] Rho, J-Y, et al., Med. Eng. Phys., 20, 92 (1998)

[18] Farr, R., et al., EPL, 84, 14001 (2008)

[19] Rayneau-Kirkhope, D. et al., EPL, 9334002 (2011)

[20] Rayneau-Kirkhope, D. et al., Phys. Rev. E, 187 (2013)

[21] Farr, R., Phys. Rev. E, 76, 046601 (2007)

[22] Banerjee, S., Mech. Mat., 72, 19 (2014)

[23] Rayneau-Kirkhope, Mech. Res. Comms., 4641 (2010)
[24] Bhat, T., et al., SAMPE J., 25, 43 (1989)

[25] The limit of the hierarchical structure presented here is fractal and exhibits a fractal dimension as calculated in Rayneau-Kirkhope, D., et al., Phys. Rev. E, 87, 063204 (2013)

[26] Elsayed, M., et al., Mechanics of Materials, 42, 709 (2010)

[27] Rayneau-Kirkhope, D., et al., Phys. Rev. Lett. 109 204301 (2012)

[28] Euler L., Mem. Acad. Sci. Berlin, 13, 252 (1759)

[29] Timoshenko S. P. and Gere J. M, Theory of Elastic Stability, (McGraw Hill, 1986)

[30] Koiter, W. T., Ph.D. thesis, Technische Hogeschool Delft, 1945.

[31] Landau, L. D. and Lifshitz, E. M., Theory of Elasticity, Pergamon Press, 1959.

[32] Sun, H., et al., Adv. Mat., 252554 (2013)

[33] Hu, N., et al, Int. J. Solids Struct., 446535 (2007)

[34] Shima, H., Materials, 5, 47 (2012)

[35] Benson, E., et al., Nature, 523441 (2015)

[36] Goodman, R., et al., Science, 3101661 (2005)

[37] Schiffels, D. et al., ACS Nano, 7, 6700 (2013) 